\title{
Effects of Shenfu injection on shivering prevention and hemodynamic impact in elderly patients underwent transurethral resection of the prostate
}

\author{
Weiqi $\mathrm{KE}^{1}$, Yuting WANG ${ }^{1}$, Xukeng GUO ${ }^{1 * \# ~(i D), ~ X u a n ~} \mathrm{JI}^{2 * \#}$, Shaohui ZHUANG
}

\begin{abstract}
We aim to investigate the effect of Shenfu injection before anesthesia on shivering and its impact on hemodynamic parameters. Eighty patients underwent transurethral resection of the prostate were randomized and received $50 \mathrm{~mL}$ Shenfu injection (experiment group) or $50 \mathrm{~mL} 0.9 \%$ sodium chloride injection (control group) before anesthesia. Incidence of shivering, axillary temperature, heart rate, systolic and diastolic blood pressure were monitored before, during, and after surgery. Compared to the control group, patients in the experiment group had significantly less decrease in temperature $\left(36.02 \pm 0.29^{\circ} \mathrm{C}\right.$ in the experiment group versus $35.50 \pm 0.33{ }^{\circ} \mathrm{C}$ in the control group, $\left.\mathrm{p}=0.047\right)$. Patients in the experiment group had lower incidence of shivering $(10 \%$ in the experiment group versus $27.5 \%$ in the control group, $\mathrm{p}=0.042$ ). On average, patients in the experiment group had significantly more stable hemodynamic parameters: with lower heart rate, less decrease in systolic and diastolic blood pressure $(\mathrm{p}<0.05$ for all). No significant difference in adverse events were observed. Our study demonstrated that among elderly patients who received transurethral resection of prostate under combined spinal and epidural anesthesia, Shenfu injection before anesthesia prevented postoperative shivering and reduced the temperature and hemodynamic perturbation of anesthesia and surgery.
\end{abstract}

Keywords: anesthesia; hemodynamic; Shenfu injection; shivering.

Practical Application: Perioperative care for shivering prevention.

\section{Introduction}

Shivering is a common complication of anesthesia with incidence rate reported to be between $26 \%$ and $74 \%$ in the general anesthesia (Eberhart et al., 2005). Clinically, shivering is noticed as uncoordinated and involuntary oscillatory contraction of skeletal muscle to elevate body's temperature. Shivering might be associated with several adverse events to both healthy individuals and hospitalized patients who are recovering from anesthesia or treated with hypothermia (Park et al., 2012). Shivering is uncomfortable to patients (Ostheimer \& Datta, 1981). It might also worsen post operation pain by stretching surgical incisions (Crossley, 1992). In addition, shivering increases consumption of oxygen, production of carbon dioxide, and release of catecholamine, which result in increased cardiac output, arterial pressure and heart rate (Bicer et al., 2006). These conditions might impose extra risk on patients with existing cardiac or pulmonary conditions. Effective prevention and treatment of shivering are essential in increasing postoperative comfort and reducing shivering related complications.

With an aging population, nowadays, surgeries among elderly population is more and more common. These patients are more likely to have complications of heart, brain and lung, which might impose extra challenges for anesthesia and operation. In transurethral resection of the prostate (TURP), the average age of patients undergoing TURP is approximately 69 years. Intraspinal anesthesia is commonly performed. Intraspinal anesthesia might result in skin vessels dilation in the blockage area, causing local heat redistribution and decrease central temperature. In addition, elder patients might have reduced thermoregulatory ability, which further increase the risk of shivering. In TURP operation, a large amount of fluid flushing is often required and intraoperative bleeding might occur. Altogether, patients might be at risk of low blood pressure, low body temperature during operation, which all increase the risk of intraoperative and postoperative shivering and unstable hemodynamic status.

Shenfu injection solution, a traditional Chinese medicine, has been used to improve cardiac function, improve the tolerance to anesthesia and surgery, and stabilize hemodynamic parameters (Wen-Ting et al., 2012; Ji et al., 2011; Gu et al., 2012). Shenfu injection might have a beneficial effect on shivering prevention, but no study has been done. In this study, we aimed to assess the effect of Shenfu injection on intra- and post operation shivering. Our hypothesis was Shenfu injection can stabilize body temperature during TURP, reduce the incidence of shivering and stabilize the hemodynamic parameters.

\section{Participants and method}

The study is a randomized, double blind, and placebo-controlled study. The study was approved by the Institutional Review Board

${ }^{1}$ Department of Anesthesiology, The First Affiliated Hospital of Shantou University Medical College, Shantou, Guangdong Province, China

${ }^{2}$ Department of Traditional Chinese Medicine, The First Affiliated Hospital of Shantou University Medical College, Shantou, Guangdong Province, China

*Xukeng Guo and Xuan Ji first affiliated Hospital of Shantou, University Medical College.

*Corresponding authors: 396273835@qq.com, jilan93@163.com 
of our hospital. Informed consent form was signed by each patient and their families before study enrollment. Inclusion criteria were: 1) underwent transurethral resection of the prostate; 2) ASA physical status 1-3;3) underwent combined spinal and epidural anaesthesia; 4) age $>60$ years; and 5) signed informed consent. Exclusion criteria were: 1) changes in surgical and anesthesia method; 2) allergies to Shenfu injection solution; or 3 ) refused to sign the informed consent form.

Between January 2018 and December 2018, 80 patients who received transurethral resection of prostate under combined spinal and epidural anesthesia consent to the study. Baseline characteristics, including age, ASA statute, diagnosis of hypertension, diabetes or neuropathy were documented. Neuropathy was defined as any diagnosis of any neurological diseases in the central nervous system, the peripheral nervous system, or the autonomic nervous system. Cerebral infarction, Parkinson disease and Alzheimer disease were observed in enrolled participants. Patients were randomized to experiment group (50 mL Shenfu injection before anesthesia) or control group (50 $\mathrm{mL} 0.9 \%$ sodium chloride injection before anesthesia) by random number table. Forty patients were randomized to the experiment group, and 40 patients were randomized to the control group.

\subsection{Operation}

Surgery room temperature was maintained at $22-23^{\circ} \mathrm{C}$. A convective device blanket was applied to the center ventral body surface. No infusion warmer was used during the operation. Electrocardiograph (ECG), heart rate (HR), systolic blood pressure (SBP), and diastolic blood pressure (DBP) were routinely monitored. For patients in the experiment group, $50 \mathrm{~mL}$ of Shenfu injection solution was given before anesthesia. For patients in the control group, $50 \mathrm{~mL}$ of $0.9 \%$ sodium chloride injection solution was given accordingly. Combined spinal and epidural anesthesia was performed. Epidural anesthesia was performed with $0.5 \mathrm{~mL}$ $0.5 \%$ ropivacaine. The anesthesia plane was controlled at a level around $\mathrm{T} 10$. If the anesthesia plane does not meet the surgical requirements, an addition of $10-15 \mathrm{~mL}$ of $0.5 \%$ ropivacaine hydrochloride was added. If the patient experienced bradycardia, hypotension et al, the anesthesiologist would take appropriate action and record the situation.

Axillary temperature at baseline, after the anesthesia, at the beginning of the operation, and at the end of the operation was documented. Shivering was graded as a five-point grade: 0 : no shivering; 1 : fasciculation of vertical muscles or peripheral vasoconstriction but no visible tremor; 2: tremor involving one muscle group; 3: tremor involving more than one muscle group; 4: gross muscle activity involving the entire body. A nurse blinded to patient assignment documented shivering every 15 minutes after the operation start. If a grade 3 or grade 4 shivering occurred, the patient was considered to have a clinical shivering, and a slow intravenous infusion of $50 \mathrm{mg}$ tramadol was administered. Hemodynamic changes, including HR, SBP and DBP were monitored. Potential adverse reactions, including nausea, vomiting, dizziness and headache was monitored during operation and after operation.

\subsection{Statistical analysis}

Statistical analysis was performed using SPSS 22.0 statistical software (Chicago, SPSS Inc.). Continuous data were expressed as mean \pm standard deviation if following normal distribution, and expressed as median (interquartile range) if not. Categorical variables were expressed as number (percentage). Intention to treat analyses were performed using repeated measures ANOVA. Categorical data were compared using Chi-square test between groups. Two-sided $\mathrm{p}<0.05$ was considered statistically significant. The datasets used or analysed during the current study are available from the corresponding author on reasonable request.

\section{Results}

\subsection{Baseline characteristics}

A total of 80 patients undergoing transurethral resection of the prostate were included in the study. On average, patients were 73 years old. There were no significant differences in the age, ASA status, comorbidities, operation time, or intraoperative blood loss between the two groups (Table 1).

\subsection{Axillary temperature}

There was no significant difference in the baseline axillary temperature between the two groups $\left(\mathrm{T}=36.60 \pm 0.42{ }^{\circ} \mathrm{C}\right.$ in the control group, and $\mathrm{T}=36.56 \pm 0.45^{\circ} \mathrm{C}$ in the experiment group, $\mathrm{p}>0.05$, Figure 1). The body temperature of both groups decreased with the start of anesthesia and operation. The mean temperature in the control group decreased to $35.50 \pm 0.33^{\circ} \mathrm{C}$ post-operation, which was significantly lower compare to that at baseline $(\mathrm{p}=0.04)$. The mean temperature in the experiment group was also significantly lower than that at baseline $\left(\mathrm{T}=36.02 \pm 0.29^{\circ} \mathrm{C}, \mathrm{p}=0.04\right)$. However, compared to the control group, the post-operation temperature was significantly higher in the experiment group $(\mathrm{p}=0.047)$.

\subsection{Shivering}

Eleven patients (27.5\%) experienced 3-4 grade shivering in the control group, while 4 patients (10\%) experienced

Table 1. Baseline characteristics of patients.

\begin{tabular}{lcc}
\hline & Control $(\mathrm{n}=40)$ & Shenfu $(\mathrm{n}=40)$ \\
\hline Age (years) & $73.4 \pm 10.2$ & $72.6 \pm 11.6$ \\
ASA status & & \\
I & 4 & 3 \\
II & 24 & 27 \\
III & 12 & 10 \\
Hypertension & 23 & 21 \\
Diabetes & 9 & 10 \\
Neuropathy & 11 & 9 \\
Operation (min) & $68.6 \pm 15.3$ & $70.2 \pm 13.6$ \\
Intraoperative blood & $135.5 \pm 25.9$ & $129.9 \pm 29.8$ \\
loss (mL) & & \\
\hline
\end{tabular}




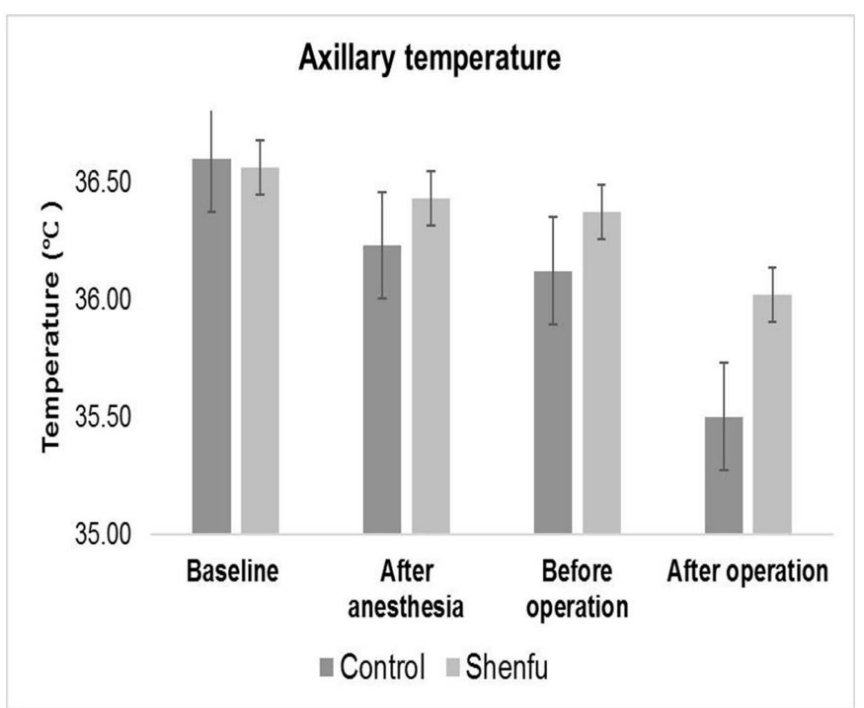

Figure 1. Axillary temperature at baseline, after anesthesia, before operation, and after operation in the control group and the experiment group. Compared to the baseline temperature, the post-operation temperature in both groups were significantly lower ( $\mathrm{p}<0.05$ for both). In addition, the post-operation temperature was significantly higher in the experiment group than that in the control group $(\mathrm{p}=0.047)$.
3 grade shivering. The incidence of $3-4$ grade shivering in the experiment group was significantly lower $(\mathrm{P}=0.042$, Table 2$)$. Not surprisingly, the total dosage of tramadol administered was significantly lower in the experiment group (200 mg in the experiment group versus 550mg in the control group, $\mathrm{p}<0.05$ ).

\subsection{Hemodynamic parameters}

There was no significant differences in the baseline HR, SBP, or DBP between the two groups. No significant difference was observed after injection of Shenfu or placebo injection. With the start of anesthesia, the HR in the control group increased significantly $(83.5 \pm 9.8 \mathrm{bpm}$ right before operation in the control group, $\mathrm{p}=0.031$ compared to the baseline) while the $\mathrm{HR}$ in the experiment decreased significantly $(76.4 \pm 7.9 \mathrm{bpm}$ right before intervention in the experiment group, $\mathrm{p}=0.027$ compared to the baseline, Figure 2A). After operation, the HR in the experiment group was significantly lower than that in the control group $(73.9 \pm 9.2$ in the experiment group versus $86.4 \pm 12.1$ in the control group, $\mathrm{p}=0.015$ ).

Blood pressure of the two groups decreased significantly after anesthesia, and further decreased after operation ( $p=0.022$ in the control group and $\mathrm{p}=0.037$ in the experiment group).
A

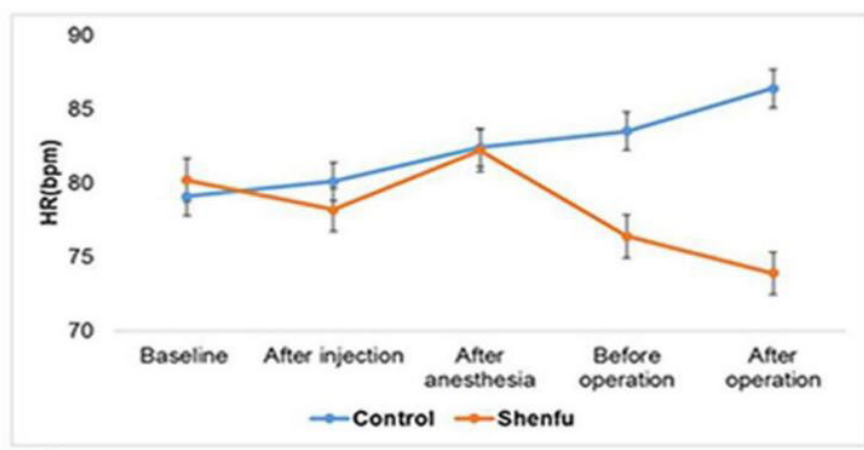

C

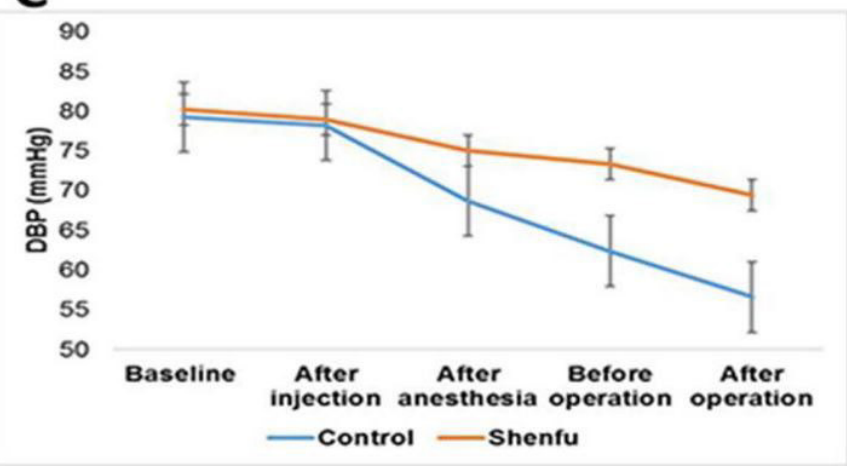

B

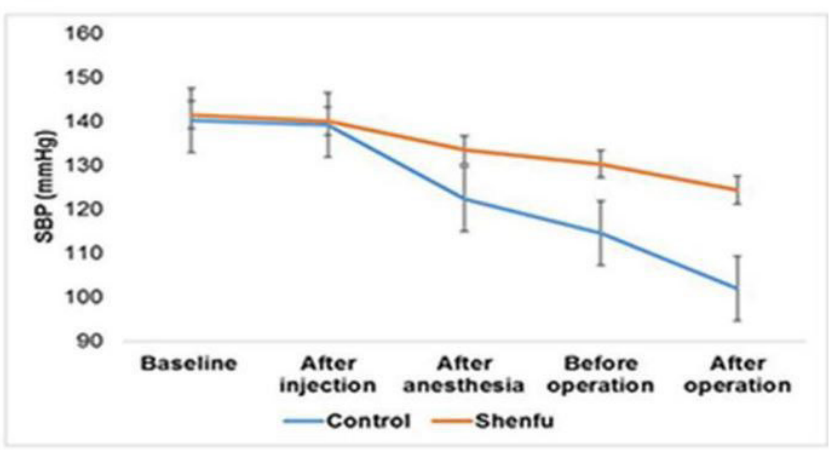

Figure 2. Change of hemodynamic data in the two groups. The heart rate (HR) in the control group increased significantly while the HR in the experiment decreased significantly (A). After operation, the HR in the experiment group was significantly lower than that in the control group. Systolic blood pressure (SBP) in the two groups decreased significantly after anesthesia, and further decreased after operation (B). Compared to the control group, SBP was significantly higher in the treatment group right before operation and after operation. Diastolic blood pressure (DBP) in the two groups decreased significantly after anesthesia, and further decreased after operation (C). Compared to the control group, DBP was significantly higher in the treatment group right before operation and after operation. 
Compared to the control group, SBP was significantly higher in the treatment group right before operation $(130.3 \pm 16.5$ in the experiment group versus $114.6 \pm 23.8 \mathrm{mmHg}$ in the control group, $\mathrm{p}=0.026$, Figure $2 \mathrm{~B})$, and after operation $(124.3 \pm 18.4$ in the experiment group versus $102.0 \pm 20.7$ in the control group, $\mathrm{p}=0.029)$. Similarly, DBP was significantly higher in the experiment group after anesthesia, right before operation and after operation ( $\mathrm{p}<0.05$ for all, Figure $2 \mathrm{C})$.

\subsection{Adverse events}

Adverse reactions including bradycardia/ tachycardia (defined as $20 \%$ deviation from baseline) and high/low blood pressure (defined as $20 \%$ deviation from baseline) were rare (Table 3). No significant difference was observed compared to the control group ( $p>0.05)$. There was no significant difference in the incidence of nausea and vomiting between the two groups $(\mathrm{P}>0.05)$.

\section{Discussion}

In summary, our study demonstrated that among patients who received transurethral resection of prostate under combined spinal and epidural anesthesia, Shenfu injection before anesthesia prevented postoperative shivering and reduced the temperature and hemodynamic perturbation of anesthesia and surgery.

Shivering is a common complication of anesthesia. Reduced thermoregulatory ability, irrigation fluid during surgery, blood loss, intravenous cold liquid and redistribution of body temperature after anesthesia might lower body's central temperature and result in shivering. Shivering is uncomfortable for patients, might impair monitoring techniques, and might increase risk of complications (Lopez, 2018). Strenuous shivering can triple oxygen consumption and increase production of carbon dioxide, which might result in demand ischemia in patients with limited pulmonary or cardiac reservation (Kang et al., 2019; Wada et al., 2019). Prevention of intra- and post operation shivering is of great importance to the elderly, especially elderly patients with reduced cardiopulmonary function.

Two Chinese herbal medicines, Radix Ginseng (ginseng) and Radix Aconiti Lateralis Preparata (prepared aconite root) have been used to treat heart failure for over 2000 years. Clinical practice has shown that ginseng and prepared aconite root could improve patients' cardiac function and alleviate heart failure associated symptoms. Shenfu injection, which are mainly composed of extraction of ginsenosides and higenamine, has been used to treat cardiac disease in China for long period. Research has demonstrated that ginsenosides might improve ischemic myocardial metabolism, protect myocardial ultrastructure reduce calcium overload and reduce free radicals (Wen-Ting et al., 2012). Higenamine has been shown to improve myocardial contractility, improve circulation and decrease the impact of acute myocardial ischemia (Gu et al., 2012). In mice model, Shenfu injection was shown to resist the lowering of limb temperature when the mice are in endotoxin induced microcirculation disturbance state (Yang et al., 2003). Consistent with the literature, in our study, patients who received Shenfu injection before anesthesia had higher body temperature. In addition, significant prevention of shivering was observed, which might be a result of more stable body temperature. Higher blood pressure and lower heart rate intra-and post operation were observed, which might be a result of less shivering. However, the stable hemodynamic parameters might decrease the risk of shivering in return.

Effective treatment of shivering has become imperative with increasing awareness of the potential complications associated with shivering (Buggy \& Crossley, 2000). Most treatment for shivering are empiric. Unfortunately, the overall quality of the anti-shivering guidelines is low. Common pharmacological therapies include opioids, a2-agonists, anticholinergics, central nervous system stimulants, and corticosteroids (Lopez, 2018). However, potential adverse effects of these medications limit their use in clinical settings. These treatment might interfere with clinical impact during anesthesia recovery, might impede neurologic exams (Park et al., 2012). In addition, these medications might affect hemodynamic and neurologic state, especially in elderly patients. Our study suggested a potential effective pharmacological prevention strategy for shivering and we showed that it is safe among elderly population.

Our study has several limitations. First, our study is a single-center study with small sample size. Second, though the enrolled patients were old, they had no coronary disease. Larger studies in patients with higher cardiovascular risk population are needed to further validate our results.

Table 2. Shivering during and post-operation in the two groups.

\begin{tabular}{|c|c|c|c|c|c|c|}
\hline \multirow{2}{*}{ Grade } & \multicolumn{3}{|c|}{ No. clinical shivering } & \multicolumn{2}{|c|}{ Clinical shivering } & \multirow{2}{*}{$\begin{array}{l}\text { Total dosage of } \\
\text { tramadol (mg) }\end{array}$} \\
\hline & 0 & 1 & 2 & 3 & 4 & \\
\hline Control & 4 & 15 & 10 & 8 & 3 & 550 \\
\hline Shenfu & 7 & 21 & 8 & 4 & 0 & 200 \\
\hline$P$ value & & & 0.042 & & & \\
\hline
\end{tabular}

Table 3. Incidence of adverse events in the two groups.

\begin{tabular}{lccccc}
\hline & Tachycardia & Bradycardia & Hypotention & Hypertension & Nausea and vomiting \\
\hline Shenfu & $3(7.5)$ & $1(2.5)$ & $6(15.0)$ & $3(7.5)$ & $8(20.0)$ \\
Control & $5(12.5)$ & $2(5.0)$ & $8(20.0)$ & $4(10.0)$ & $10(25.0 \%)$ \\
\hline
\end{tabular}


In conclusion, our study demonstrated that Shenfu injection before anesthesia is effective in preventing shivering and stabilizing hemodynamic parameters in patients underwent TRUP. Our result suggested that Shenfu injection could be a safe and effective therapy for shivering prevention in elderly population.

\section{Acknowledgements}

This study was supported by Scientific research project of the Traditional Chinese Medicine Foundation of Guangdong Province [2018] No. 1).

\section{References}

Bicer, C., Esmaoglu, A., Akin, A., \& Boyaci, A. (2006). Dexmedetomidine and meperidine prevent postanaesthetic shivering. European Journal of Anaesthesiology, 23(2), 149-153. http://dx.doi.org/10.1017/ S0265021505002061. PMid:16426470.

Buggy, D. J., \& Crossley, A. (2000). Thermoregulation, mild perioperative hypothermia and post-anaesthetic shivering. British Journal of Anaesthesia, 84(5), 615-628. http://dx.doi.org/10.1093/bja/84.5.615. PMid:10844839.

Crossley, A. W. (1992). Six months of shivering in a district general hospital. Anaesthesia, 47(10), 845-848. http://dx.doi.org/10.1111/j.1365-2044.1992. tb03143.x. PMid:1443474.

Eberhart, L. H., Döderlein, F., Eisenhardt, G., Kranke, P., Sessler, D. I., Torossian, A., Wulf, H., \& Morin, A. M. (2005). Independent risk factors for postoperative shivering. Anesthesia and Analgesia, 101(6), 1849-1857. http://dx.doi.org/10.1213/01.ANE.0000184128.41795. FE. PMid:16301273.

Gu, W., Li, C., Yin, W., Guo, Z., Hou, X., \& Zhang, D. (2012). Shenfu injection reduces postresuscitation myocardial dysfunction in a porcine model of cardiac arrest by modulating apoptosis. Shock (Augusta, Ga.), 38(3), 301-306. http://dx.doi.org/10.1097/ SHK.0b013e31825f6632. PMid:22683733.
Ji, X. F., Yang, L., Zhang, M. Y., Li, C. S., Wang, S., \& Cong, L. H. (2011). Shen-fu injection attenuates postresuscitation myocardial dysfunction in a porcine model of cardiac arrest. Shock (Augusta, Ga.), 35(5), 530-536. http://dx.doi.org/10.1097/SHK.0b013e31820e2058. PMid:21263380.

Kang, P., Park, S. K., Yoo, S., Hur, M., Kim, W. H., Kim, J. T., \& Bahk, J. H. (2019). Comparative effectiveness of pharmacologic interventions to prevent shivering after surgery: a network meta-analysis. Minerva Anestesiologica, 85(1), 60-70. http://dx.doi.org/10.23736/S03759393.18.12813-6. PMid:30226340.

Lopez, M. B. (2018). Postanaesthetic shivering-from pathophysiology to prevention. Romanian journal of anaesthesia and intensive care, 25(1), 73-81. http://dx.doi.org/10.21454/rjaic.7518.251.xum. PMID: 29756066 .

Ostheimer, G. W., \& Datta, S. (1981). Observations in the postpartum recovery room after various local anesthetic techniques. Regional Anesthesia and Pain Medicine, 6, 13-17.

Park, S. M., Mangat, H. S., Berger, K., \& Rosengart, A. J. (2012). Efficacy spectrum of antishivering medications: meta-analysis of randomized controlled trials. Critical Care Medicine, 40(11), 3070-3082. http:// dx.doi.org/10.1097/CCM.0b013e31825b931e. PMid:22890247.

Wada, K., Masamune, T., Ino, H., Ueda, K., Ishiyama, T., Sessler, D. I., \& Matsukawa, T. (2019). Severe hypoglycemia reduces the shivering threshold in rabbits. BMC Anesthesiology, 19(1), 126. http://dx.doi. org/10.1186/s12871-019-0794-7. PMid:31288741.

Wen-Ting, S., Fa-Feng, C., Li, X., Cheng-Ren, L., \& Jian-Xun, L. (2012). Chinese medicine shenfu injection for heart failure: a systematic review and meta-analysis. Evidence-Based Complementary and Alternative Medicine, 2012, 713149. http://dx.doi.org/10.1155/2012/713149. PMid:22611430.

Yang, F., Zheng, Y., Li, D., \& Deng, W. (2003). Effect of shenfu injection on microcirculation. Sheng wu yi xue gong cheng xue za zhi= Journal of biomedical engineering= Shengwu yixue gongchengxue zazhi, 20(1), 91-94, 100. PMID: 12744172 . 